A. Pramesh Rao, G. Swarup and Gopal-Krishna, eds.

\title{
Giant Radio Sources: Evolution and GMRT Observations
}

\author{
C.H. Ishwara-Chandra and D.J. Saikia \\ National Centre for Radio Astrophysics, TIFR, Post Bag No. 3, \\ Ganeshkhind, Pune 411007, India
}

\begin{abstract}
We study the evolution of giant radio sources and show that they are less luminous than the smaller-sized sources, and are roughly consistent with the evolutionary scenarios where the smaller-sized sources evolve to giants undergoing radiative and expansion losses. InverseCompton radiation losses due to the microwave background radiation dominates over the synchrotron losses for giant sources, while for the smaller sources, the latter dominates. We show that giant radio galaxies and quasars are consistent with the unified scheme, and their large sizes are not due to stronger cores. We also present preliminary GMRT images of three giant sources.
\end{abstract}

\section{Introduction}

The giant radio sources (GRSs), defined to be those with a projected linear size $\geq 1 \mathrm{Mpc}\left(\mathrm{q}_{\mathrm{o}}=0.5\right.$ and $\left.\mathrm{H}_{\mathrm{o}}=50 \mathrm{~km} \mathrm{~s}^{-1} \mathrm{Mpc}^{-1}\right)$, are believed to be the older counter-parts of double-lobed radio sources with smaller linear sizes. The giant sources, about 100 of which are presently known following the identifications from WENSS (cf. Ishwara-Chandra \& Saikia 1999, hereinafter referred to as IC99; Schoenmakers 1999), are useful for studying the evolution of radio sources and also probing the intergalactic medium at different redshifts. In the course of our study of a sample of Molonglo radio sources, we found two new giant quasars, increasing the total number of giant quasars to five (IC99). One of these has the highest redshift among known giant quasars. In this paper we summarize some of our results on the evolution of giant radio sources, their consistency with the unified scheme, and also present radio images of 3 giant sources with the GMRT.

\section{Evolution of giant radio sources}

The power-size or P-D diagram, which is a useful tool for studying the evolution of radio sources, for the sample of about 50 giants from IC99 and all 3CR sources with $50 \mathrm{kpc}<\mathrm{D}<1 \mathrm{Mpc}$ is shown in Figure 1 (left panel). The clear deficiency of giants of high radio luminosity suggests that the luminosity of radio sources decreases as they evolve. The evolutionary tracks suggested by Kaiser et al. (1997), which are shown in the figure, are roughly consistent with the data. However, in the models developed by Blundell, Rawlings \& Willott (1999), the luminosity declines more rapidly than the Kaiser et al. tracks, providing a somewhat better fit to the upper envelope for large linear sizes. There is also a 

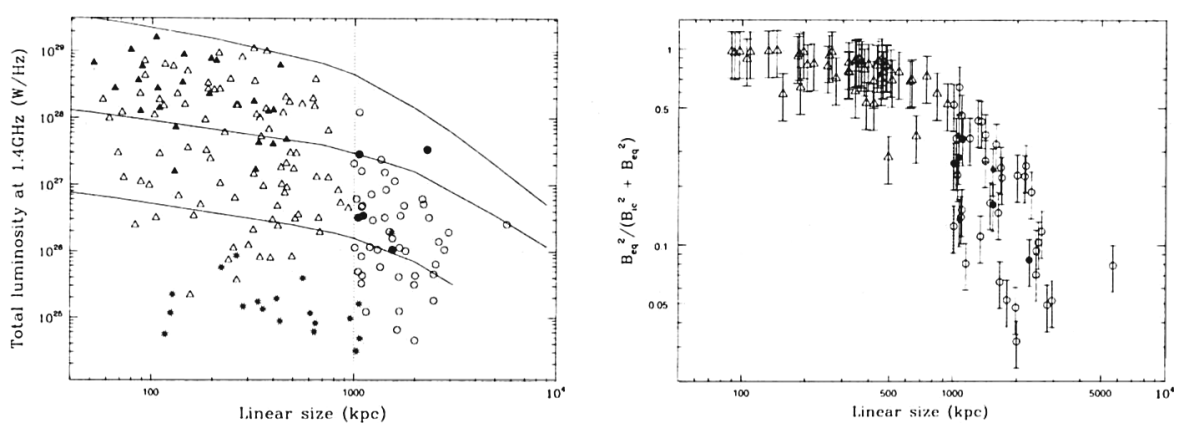

Figure 1. The projected linear size, D, is plotted against radio luminosity, $\mathrm{P}$, (left panel) and the ratio $\mathrm{B}_{e q}^{2} /\left(\mathrm{B}_{i c}^{2}+\mathrm{B}_{e q}^{2}\right)$ (right panel) for the sample of giants from IC98 and all 3CR sources with $50 \mathrm{kpc}<\mathrm{D}$ $<1 \mathrm{Mpc}$. The giant quasars and galaxies are shown by filled and open circles respectively; while the $3 \mathrm{CR}$ quasars and galaxies are shown by filled and open triangles respectively, except for those with a clear FRI structure, which are marked with an asterisk. The evolutionary scenarios for sources with jet powers of $1.3 \times 10^{40}, 1.3 \times 10^{39}$ and $1.3 \times 10^{38} \mathrm{~W}$ from Kaiser et al. (1997) are shown superimposed on the P-D diagram.

clear dearth of giants larger than about $3 \mathrm{Mpc}$. To investigate whether these large sources are missed due to selection effects, low-frequency surveys with higher sensitivity to diffuse, low-brightness emission are required. Low-frequency radio telescopes such as the GMRT could play an important role for studying the late stages of radio source evolution.

The luminosity of radio sources decrease due to expansion and radiative losses, due to both synchrotron and inverse-Compton radiation, as they evolve to large sizes. The plot of the projected linear size against the ratio $B_{e q}^{2} /\left(B_{i c}^{2}+\right.$ $\mathrm{B}_{e q}^{2}$ ), which represents the ratio of the energy loss due to synchrotron radiation to the total energy loss due to both inverse-Compton and synchrotron processes is shown in the right panel of Figure 1. It shows clearly that the radiation loss due to inverse-Compton scattering dominates over synchrotron losses for the giant radio sources. This also illustrates that inverse-Compton losses are likely to severely constrain the number of GRSs at high redshifts since the microwave background energy density increases as $(1+z)^{4}$.

\section{Constraints on Orientation and environment}

We have examined the possibility that GRSs may have evolved to these large sizes because of powerful nuclear engines (e.g. Gopal-Krishna, Wiita \& Saripalli 1989). Although many of the giants may appear to have more prominent cores than 3CR FRII sources one has to take account of an inverse correlation between the degree of core prominence and the total radio luminosity. We find that GRSs have similar core strengths to those of the smaller sources when matched in redshift or extended radio luminosity, implying that GRSs are similar objects to the normal radio sources except for being larger and perhaps older. 

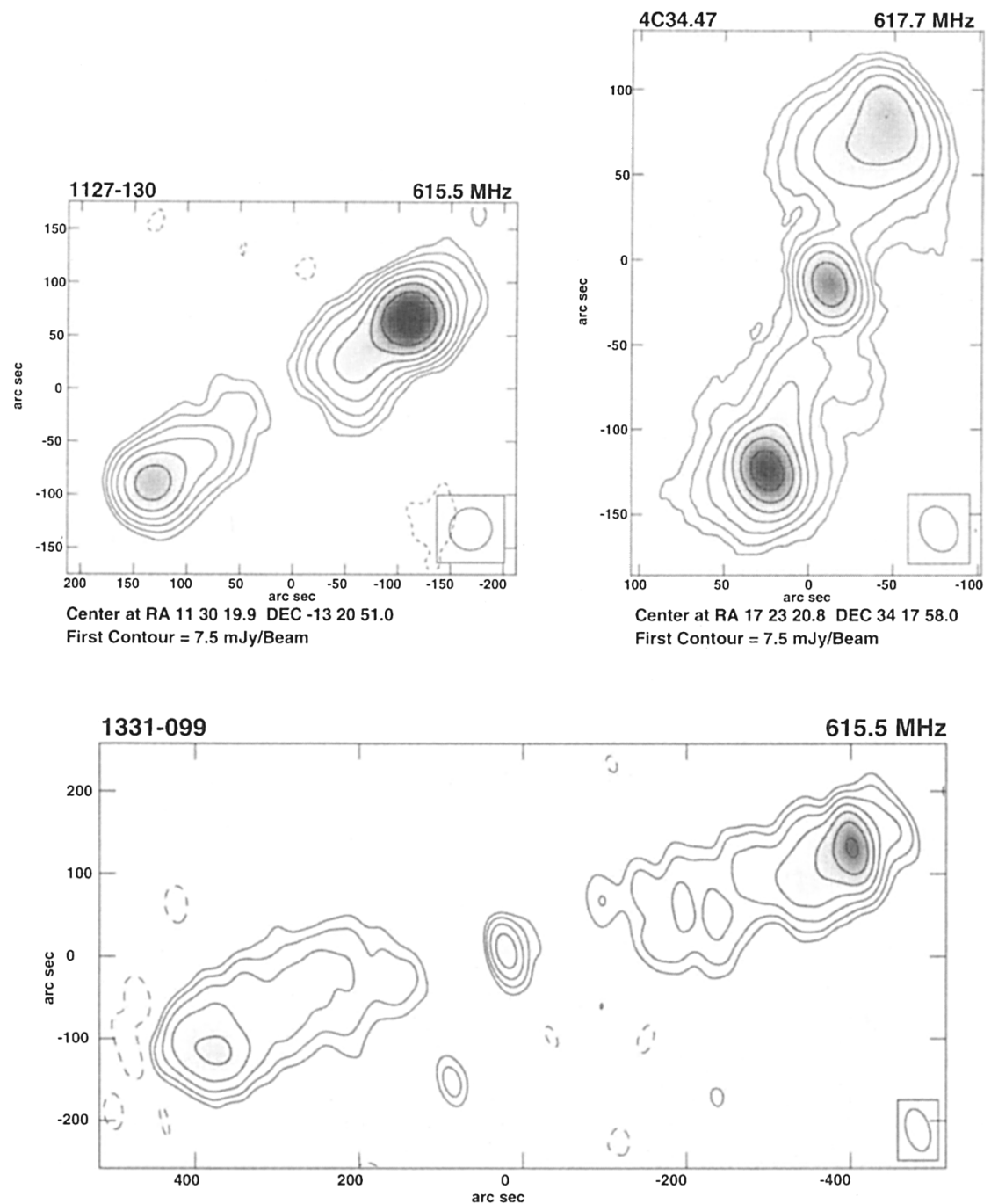

Center at RA 133418.6 DEC -10 0928.7

First Contour $=10 \mathrm{mJy} / \mathrm{Beam}$

Figure 2. Radio images of three giant radio sources made with the GMRT. The contour levels are $-2,-1,1,2,4,8, \ldots$. times the first contour, indicated below each figure. The positions of the centres in epoch J2000 are also listed. The frequency of the observations is indicated in each image. The restoring beams are $40.4^{\prime \prime} \times 39.6^{\prime \prime}$ along PA $-65^{\circ}$ for $1127-130,27.6^{\prime \prime} \times 21.5^{\prime \prime}$ along PA $24^{\circ}$ for $4 \mathrm{C} 34.47$ and $52.6^{\prime \prime} \times 28.4^{\prime \prime}$ along PA $15^{\circ}$ for $1331-099$. 
We have checked the consistency of the GRSs with the unified scheme for radio galaxies and quasars, by comparing some of the orientation-dependent features such as core strength and core variability of giant radio galaxies and giant quasars. Although the available data are limited, these properties are consistent with the unified scheme. We have also examined the environments around GRSs using their arm-length ratio and the misalignment angle. The arm-length ratio for GRSs are similar to those for the smaller sources, possibly even marginally larger, indicating that the environment could be asymmetric on Mpc scales. The misalignment angle for the GRSs is similar to that of the smaller sources, suggesting that their large sizes are unlikely to be due to a steadier ejection axis.

\section{GMRT observations of giants}

Sensitive observations of the lobes and bridges at low radio frequencies would be useful to put better constraints on the evolution of giant sources as well as for probing the intergalactic medium. Preliminary GMRT images of three giant radio sources at $\sim 615 \mathrm{MHz}$ are presented in Figure 2.

1127-130: This is the largest known giant quasar (Bhatnagar, GopalKrishna \& Wisotzki 1998), and has a redshift of 0.6337 . The integrated flux density of the entire source is $2.33 \mathrm{Jy}$, while that of the northern and southern lobes are 1.84 and $0.54 \mathrm{Jy}$ respectively. The non-detection of the radio core at $49 \mathrm{~cm}$ suggests that the core has an inverted spectrum.

1331-099: This is a low-redshift $(\mathrm{z}=0.081)$ giant radio galaxy with a prominent core. The total flux density of the source is $3.56 \mathrm{Jy}$, while that of the eastern and western lobes are 1.31 and $2.2 \mathrm{Jy}$ respectively. The core, whose flux density at $615.5 \mathrm{MHz}$ is $0.133 \mathrm{Jy}$, has a spectral index of about 0.2 between 49 and $2.8 \mathrm{~cm}$ (Saripalli et al. 1996).

1721+343: This giant quasar has a prominent jet pointing towards the southern lobe, and a strong, superluminal core (Riley \& Warner 1991; Barthel et. al 1989), which also shows evidence of variability. The present observations clearly show the bridge connecting the two lobes and the core. The flux density of the entire source is $2.57 \mathrm{Jy}$, that of the northern and southern lobes are 0.81 and $1.24 \mathrm{Jy}$ respectively while the core has a flux density of $0.5 \mathrm{Jy}$.

\section{References}

Barthel P. D. et al. 1989, ApJ, 336, 601

Bhatnagar S., Gopal-Krishna, Wisotzki L., 1998, MNRAS, 299, L25

Blundell K. M., Rawlings S., Willott C. J., 1999, AJ, 117, 677

Gopal-Krishna, Wiita P.J., Saripalli L., 1989, MNRAS, 239, 173

Ishwara-Chandra C. H., Saikia D. J., 1999, MNRAS, 309. 100 (IC99)

Kaiser C. R., Dennett-Thorpe J., Alexander P., 1997, MNRAS, 292, 723

Riley J. M., Warner P. J., 1990, MNRAS, 246, L1

Saripalli L. et al. 1996, A\&A, 306, 708

Schoenmakers, A. P., 1999, Ph. D.Thesis, University of Utrecht 